\title{
Ultrabroadband generation of multiple concurrent nonlinear coherent interactions in random quadratic media
}

\author{
Luis Mateos, ${ }^{1}$ Pablo Molina, ${ }^{1}$ Juan F. Galisteo-López, ${ }^{2}$ Cefe López, ${ }^{2}$ Luisa E. Bausá, ${ }^{1}$ \\ and Mariola O Ramírez ${ }^{1, a)}$ \\ ${ }^{1}$ Dpto. Física de Materiales and Instituto Nicolás Cabrera, Universidad Autónoma de Madrid, 28049 Madrid, \\ Spain \\ ${ }_{2}^{2}$ Instituto de Ciencia de Materiales de Madrid (CSIC), Calle Sor Juana Inés de la Cruz, 28049 Madrid, Spain
}

(Received 1 July 2013; accepted 19 August 2013; published online 3 September 2013)

\begin{abstract}
Triply coincident nonlinear second harmonic interactions continuously tunable in an extremely large spectral range $(800-1400 \mathrm{~nm})$ are demonstrated in strontium barium niobate random quadratic crystal. The spectral dependence of the triple process is in agreement with a first order quasi-phasematching collinear interaction. The analysis of the polarization states of the generated beams reveals mutually coherent interactions between different processes simultaneously contributing to the total intensity. The results constitute a necessary step in the implementation of ultra-broadband entangled photon pairs from randomly poled structures and are relevant to a wide range of applications, for which broadband polarization controlled light is required. (C) 2013 AIP Publishing LLC.

[http://dx.doi.org/10.1063/1.4819855]
\end{abstract}

Ferroelectric crystals exhibiting random distribution of alternate domains are increasingly employed in nonlinear optics to design compact multi-wavelength light sources, since they allow for the simultaneous phase matching of several nonlinear processes. In these systems, the generation of multiple nonlinear interactions, as well as their tunable response, occurs at the expense of the efficiency. However, their simplicity, compared to other nonlinear tunable devices based on more sophisticated superstructures of inverted domains, makes them attractive sources for the development of advanced multi-photon devices. ${ }^{1-7}$ Substantially less explored is the capability of these systems to manipulate and control the polarization state of the generated nonlinear beams in a broad frequency range. The simultaneous presence of two or more different phase matching processes, involving different polarization states, is of interest in many applications. Polarization-entangled photon pairs, useful for quantum computing and communications, ${ }^{8}$ polarization-free frequency converters, valuable for laser fibers and optical communications, ${ }^{9}$ or all optical devices, including all optical isolators or polarization switchers, ${ }^{10,11}$ are some examples of optical devices that could take advantage of concurrent nonlinear phase-matching processes in a single crystal. Moreover, multipartite quantum entanglement or the possibility to obtain quantum correlation without interferometers have been proposed when a set of three or more photons with different polarization states are simultaneously phase-matched in an optical parametric oscillator. ${ }^{12,13}$ In this context, triply concurrent nonlinearities, namely, type 0 , type I, and type II second harmonic generation ( $\mathrm{SHG}$ ), have been demonstrated in a carefully designed multi-grating periodically poled $\mathrm{KTiOPO}_{4}$ crystal, after accurate wavelength and temperature control. ${ }^{14}$ The complexity in the sample preparation and experimental set-up can be strongly

\footnotetext{
${ }^{a)}$ Electronic mail: mariola.ramirez@uam.es. Tel.: +34-914976428. Fax: $+34-914978579$.
}

simplified by using quadratic random nonlinear media as nonlinear optical sources. In this letter, we have focused our attention on as-grown congruent Strontium Barium Niobate (SBN) crystal due to its large nonlinear coefficients and to the presence of a random distribution of ferroelectric domains. ${ }^{2,4}$ Up to three different coincidental quadratic nonlinear parametric interactions, with different input/output polarization states, are experimentally demonstrated to occur in a broad spectral range covering the whole visible spectrum. These three SHG processes are simultaneously obtained at room temperature and involve a single fundamental beam and first order quasi-phase matching (QPM) collinear interactions. Furthermore, a coherent superposition of different parametric processes is demonstrated by means of the SHG polar plot analysis.

SBN belongs to the $4 \mathrm{~mm}$ symmetry group. Accordingly, for a fundamental beam propagating perpendicular to the ferroelectric $\mathrm{z}$-axis, the non-zero components of the quadratic nonlinear susceptibility tensor, $\chi^{(2)}$, allows up to three different concurrent $\mathrm{SH}$ interactions with the same frequency, but with different input/output polarization states. The three simultaneous nonlinear interactions are eee (type 0), ooe (type I), and oeo/eoo (type II), $e$ and $o$ being the extraordinary and ordinary polarization, respectively. The two first letters denote the fundamental beam polarization, while the third one stands for the second harmonic polarization. In a collinear configuration, the coherence length required to QPM the allowed SHG processes can be written as

$$
l_{c}=\frac{\Lambda}{2}=\frac{\lambda}{2}\left(2 n_{2 \omega}^{o, e}-n_{\omega}^{o, e}-n_{\omega}^{o, e}\right)^{-1},
$$

where $n^{o, e}$ refers to the ordinary/extraordinary refractive index of SBN at a given frequency and $\lambda$ is the pump fundamental wavelength. This coherence length has been determined for the three different possible QPM processes, and it is illustrated in Fig. 1(a). For the calculations the refractive 


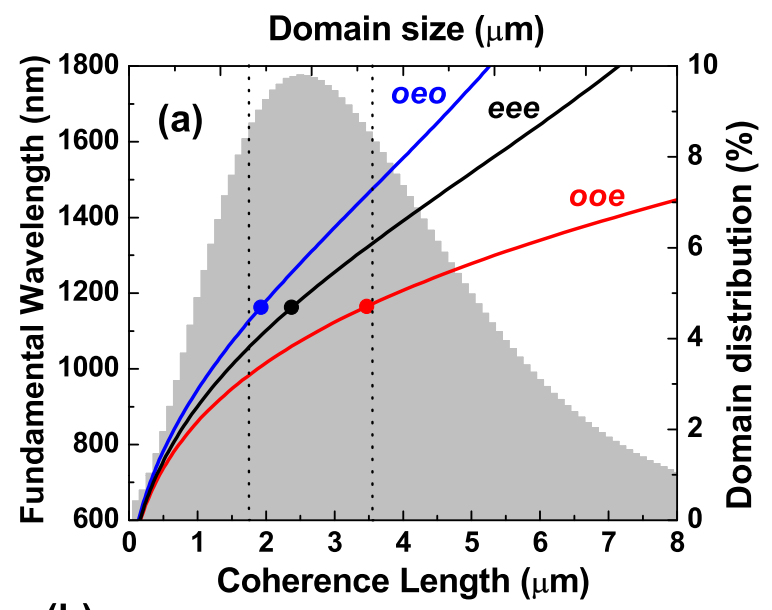

(b)

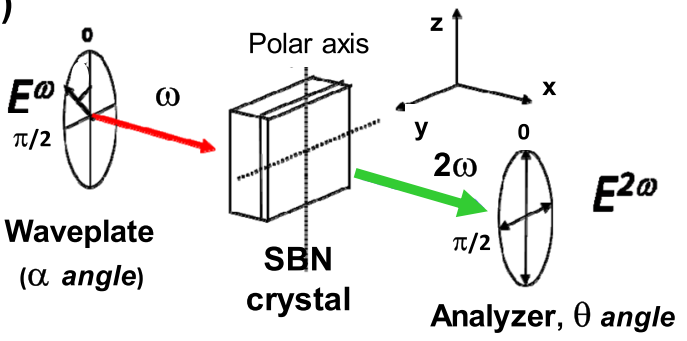

FIG. 1. (a) Spectral dependence of the coherence length in a collinear configuration for the three possible QPM collinear processes in SBN. Red, black, and blue lines correspond to ooe, eee, and oeo type processes, respectively. The coherent lengths for triple QPM-SHG for a fundamental wavelength tuned at $1.06 \mu \mathrm{m}$ are marked with dots. The gray region represents the domain size distribution in as-grown SBN. Dashed lines delimit the range of maximum efficiency. (b) Experimental set-up diagram.

index values given by Woike et al. have been employed. ${ }^{15}$ The gray region in Fig. 1(a) represents the domain size distribution in the studied SBN crystal. As observed, the size of the as-grown ferroelectric domains spreads from $0.1 \mu \mathrm{m}$ up to $\sim 10 \mu \mathrm{m}$, providing a variety of quasi-phase matching conditions over a large spectral range for each independent polarization process. Nonetheless, the generated SHG response depends on the number of domains, and it is modulated by the value of the non-zero nonlinear $d_{i j}$ component involved in the process. In our system, the most frequent domain size and variance was found to be around $2.5-3 \mu \mathrm{m}$ and $1.8 \mu \mathrm{m}$, respectively. Thus, SHG-QPM processes in the ranges $800-1300 \mathrm{~nm}, 800-1400 \mathrm{~nm}$, and $800-1600 \mathrm{~nm}$ for the ooe, eee, and oeo parametric processes, respectively, can be expected. Note that for the type-0 process, QPM-SHG in SBN has been reported by involving domain sizes which only represent $\sim 2 \%$ of the total distribution. ${ }^{2}$ Furthermore, on this basis, a triply coincident QPM configuration for the three symmetry allowed parametric (polarization) processes can be predicted in the $800-1300 \mathrm{~nm}$ spectral range, its maximum being centered around $1 \mu \mathrm{m}$. As a representative example, the required coherent lengths to obtain triple QPMSHG for a fundamental wavelength of $1.06 \mu \mathrm{m}$ have been marked with solid dots in Fig. 1(a). As seen, they all lie within the 2-3.7 $\mu \mathrm{m}$ domain size range, which represents $9 \%$ of the maximum in the domain distribution (marked with dashed lines in the figure). Therefore, in addition to the simultaneous generation of three different parametric processes, a continuously tunable broad spectral range in which the three processes coexist can be expected for a single SBN random quadratic medium.

In order to assess the ability of the system as a nonlinear broadband source for which the polarization state can be tailored, we carried out two different and complementary experiments: (i) a polarization analysis of the generated $\mathrm{SH}$ response for a fundamental incident beam at a fixed frequency and (ii) the determination of the tunning range of the SHG under different polarization geometries. As excitation sources, a ps-laser at $1064 \mathrm{~nm}$ (Spectra Physics Navigator ${ }^{\circledR}$ ) or a tunable optical parametric amplifier (Coherent OPerASolo $^{\circledR}$ ) generating $140 \mathrm{fs}$ pulses at a repetition rate of $1 \mathrm{kHz}$ in the $800-1400 \mathrm{~nm}$ spectral range were employed. In all the cases, the fundamental beam travelled perpendicular to the polar axis, along the $\mathrm{X}$ crystallographic direction. The SHG radiation was emitted in the plane perpendicular to $\mathrm{Z}$, in agreement with previous works. ${ }^{15,16}$ In our experiments the SHG intensity was measured at the exit face of the crystal in a collinear configuration by using the appropriate interference filters. The eooloeo SH process was selected by setting the input linear polarization $\alpha$, at $45^{\circ}$ with respect to the ferroelectric $\mathbf{z}$-axis of the crystal. Crossed polarizes were employed to verify that there was no signal arising from other SHG processes. Notice that while the eee and ooe processes results into pure extraordinary SH signals, the ordinary polarized SHG beam obtained by means of two fundamental photons with orthogonal polarizations, i.e., eoo/oeo process, also allows for the other ooe and eee nonlinear processes to occur simultaneously. The experimental set-up diagram is shown in Fig. 1(b).

We first analyze the angular dependence of the polarization properties of the SH response by using a ps-laser tuned at $1064 \mathrm{~nm}$ as excitation source. This wavelength was chosen for being close to that of the maximum efficiency for the three nonlinear processes (see Fig. 1(a)). Two different configurations were analyzed. First, the polarization state of the generated nonlinear response was studied by rotating the analyzer counter-clockwise from $\theta=0$ to $\theta=2 \pi$ radians while keeping the linear polarization state of the incident light, $\alpha$, fixed at $\alpha=0$ and $\alpha=\pi / 4$. In a second set of experiments the intensity of the extraordinary and ordinary polarization states of the SHG radiation was studied as a function of the polarization of the incident light, $\alpha$, which was azimuthally varied counter-clockwise from 0 to $2 \pi$ radians. In all cases, the angle origin $(\theta=0$ and $\alpha=0)$ was parallel to the z-axis of the crystal. The results are shown in Fig. 2. It is clear that SHG with orthogonal polarization states can be obtained in SBN by using a single fundamental beam, in contrast to previous claims. ${ }^{16,17}$ Additionally, as highlighted in the inset of Fig. 2(c), the extraordinary SH signal is reduced to zero for a particular input polarization state, hence pointing toward mutually coherent processes and opposite sign of the involved nonlinear coefficients. ${ }^{18}$ As a further confirmation, the angular dependence of the $\mathrm{SH}$ polarization response was interpreted taking into account the non-zero components of the nonlinear tensor of SBN, which provide information not only on the input/output polarization states but also on the coherence of the different nonlinear processes. 

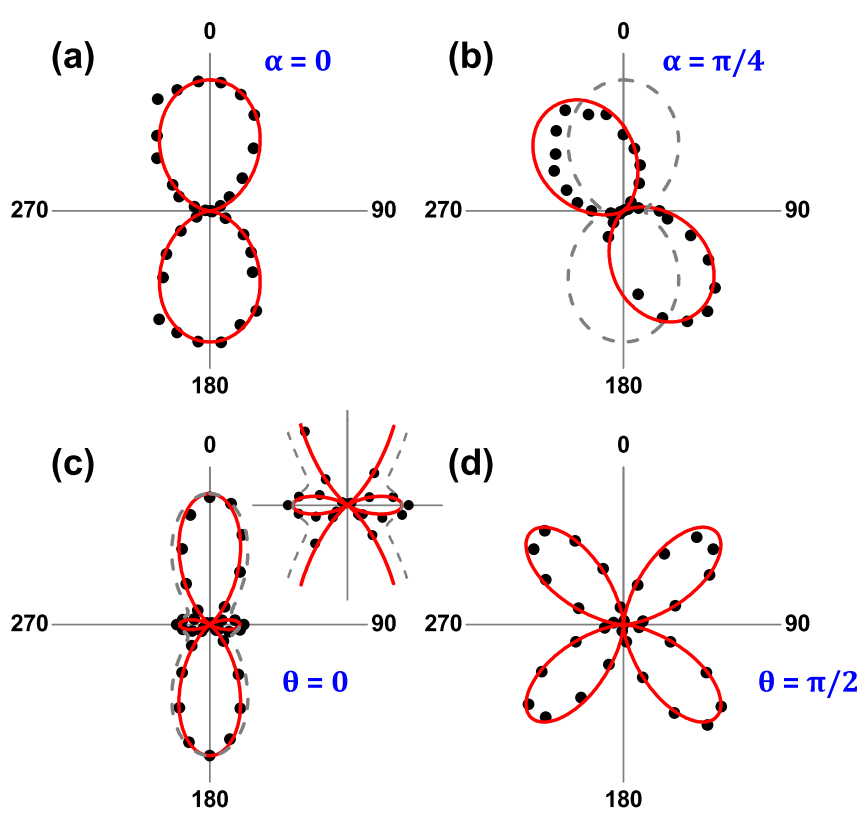

FIG. 2. Polarization of the SHG for a fundamental input beam linearly polarized at (a) $\alpha=0$ and (b) $\alpha=\pi / 4$; (c) intensity variation of the extraordinary $(\theta=0)$ and (d) ordinary $(\theta=\pi / 2)$ SHG as a function of the polarization angle of the fundamental beam. Solid lines correspond to the fits obtained from Eqs. (8)-(11). The processes corresponding to mutually incoherent interactions are plotted in dashed lines.

For a linearly polarized fundamental beam propagating along the $X$ axis, the nonlinear polarization vector for the crystal symmetry of SBN is given by

$$
\begin{gathered}
P_{x}^{(2)}=0, \\
P_{y}^{(2)}=2 d_{24} E_{o}^{2} \sin \alpha \cos \alpha, \\
P_{z}^{(2)}=\left[d_{31} \sin ^{2} \alpha+d_{33} \cos ^{2} \alpha\right] E_{o}^{2},
\end{gathered}
$$

where $\alpha$ is the polarization angle of the fundamental input beam and $d_{i j}$ the nonlinear coefficients. These equations can be written as a function of the angle $\theta$ which defines the linear polarization state of the SHG response in the following form:

$$
\begin{gathered}
P_{x}^{\prime(2)}=0 \\
P_{y}^{\prime(2)}=\left[2 d_{24} \sin \alpha \cos \alpha \sin ^{2} \theta\right. \\
\left.-\left(d_{31} \sin ^{2} \alpha+d_{33} \cos ^{2} \alpha\right) \sin \theta \cos \theta\right] E_{0}^{2} \\
P_{z}^{\prime(2)}=\left[-2 d_{24} \sin \alpha \cos \alpha \sin \theta \cos \theta\right. \\
\left.+\left(d_{31} \sin ^{2} \alpha+d_{33} \cos ^{2} \alpha\right) \cos ^{2} \theta\right] E_{0}^{2}
\end{gathered}
$$

where $E_{0}{ }^{2}=I_{0}$ represents the intensity of the fundamental beam. The intensity of the linearly polarized SHG is proportional to the nonlinear polarization, and it varies depending on the (in)-coherence of the different processes. In particular, this is reflected on the polarization dependence of the effective nonlinear coefficient of the interacting beams. ${ }^{18-20}$ The calculated coherent SHG intensity expressions for the different polarization processes displayed in Fig. 3 can be written as

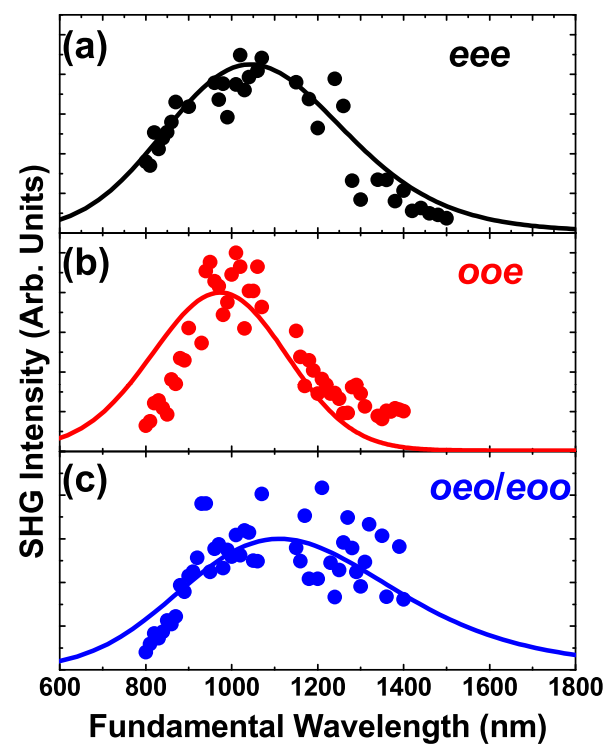

FIG. 3. Spectral dependence of the eee (type 0), ooe (type I), and oeo/eoo (type II) triply concurrent SH processes in SBN. Solid lines correspond to the calculated collinear first order QPM-SHG intensity distribution.

$$
\begin{gathered}
\alpha=0 \quad I_{T}=I_{0}^{2}\left(d_{33}^{*}\right) \cos ^{2} \theta \\
\alpha=\frac{\pi}{4} \quad I_{T}=I_{0}^{2}\left[\left(d_{24}^{*} \sin ^{2} \theta-\frac{1}{2}\left(d_{31}^{*}+d_{33}^{*}\right) \sin \theta \cos \theta\right)^{2}\right. \\
\left.+\left(-d_{24}^{*} \sin \theta \cos \theta+\frac{1}{2}\left(d_{31}^{*}+d_{33}^{*}\right) \cos ^{2} \theta\right)^{2}\right], \\
\theta=0 \quad I_{T}=I_{0}^{2}\left(d_{31}^{*} \sin ^{2} \alpha+d_{33}^{*} \cos ^{2} \alpha\right)^{2} \\
\theta=\frac{\pi}{2} \quad I_{T}=4 I_{o}^{2}\left(d_{24}^{*}\right)^{2} \sin ^{2} \alpha \cos ^{2} \alpha
\end{gathered}
$$

where $d_{i j}^{*}=\sqrt{f_{i j}(\lambda)} \times d_{i j}$ represents the involved nonlinear coefficients weighted by the distribution function of inverted domains, $f_{i j}(\lambda)$, i.e., the number of inverted domains of a certain width participating on the corresponding QPM process. Theoretical fits to the experimental polar plots based on Eqs. (8)-(11) are shown as solid lines in Fig. 2. A good agreement was obtained between the experimental and theoretical curves, thus confirming a coherent interaction among the different parametric processes. From the fitting, the relative value between the involved nonlinear coefficients was found to be $\left|\frac{d_{31}}{d_{33}}\right|=0.57,\left|\frac{d_{24}}{d_{33}}\right|=0.18$, and $\left|\frac{d_{24}}{d_{31}}\right|=0.32$, and the opposite sign between $d_{33}$ and $d_{31}$ was established. For the sake of comparison, the theoretical curves expected for the incoherent case has been plotted in dashed lines. Their corresponding theoretical expressions have not been included in the manuscript to smooth reading. It is clear that the shapes of the polar plots in Figs. 2(b) and 2(c) differ significantly, depending on whether contributions from the different processes exhibit internal interference or not. Thus, in addition to the simultaneous generation of triply concurrent nonlinear parametric processes, a coherent process between the interacting extraordinary second harmonic waves along the propagation direction is revealed. At this point we would like to mention that in contrast to the incoherent nature for the 
nonlinear process reported by Trull et al. under non-collinear dual-pump configuration, ${ }^{19}$ a coherent superposition of three parametric contributions has been recently reported for Cerenkov type SHG in SBN. ${ }^{18}$ Further, SHG speckle pattern formation by an SBN crystal has been recently demonstrated both experimentally and theoretically. ${ }^{21}$

Figure 3 shows the measured RT-SHG intensity as a function of the fundamental wavelength in the $800-1400 \mathrm{~nm}$ spectral range obtained for different linear polarization geometries: eee (type 0), ooe (type I), and oeoleoo (type II). For the sake of comparison each parametric interaction (polarization process) has been plotted in a separate panel. The theoretically obtained spectral responses for each independent polarization process are shown in solid lines. In the calculations, only first order QPM collinear interactions were considered. Additionally, the values of the non-zero nonlinear $d_{i j}$ components obtained from the polar plot analysis were weighted by the distribution function, $f_{i j}(\lambda)$, to take into account the size distribution of the ferroelectric domains participating on the frequency conversion process at a wavelength, $\lambda$. As seen, the SHG can be continuously tuned from 400 to $700 \mathrm{~nm}$ under different polarization geometries displaying a spectral dependence that correlates well with a first order QPM collinear interaction. Note that some deviations from the theoretical curve are observed possibly due to inaccuracies of the Sellmeier equation, particularly for ordinary polarized waves. These results point out the capability of random nonlinear photonic structures to generate widely tunable triply concurrent quadratic parametric interactions by using a single fundamental beam. Moreover, they are obtained at room temperature without the need of any angular or thermal adjustment of the nonlinear structure. An estimation of the conversion efficiency for the green spectral region gave a value in the order of $0.001 \%$ for the eee process. $^{2}$ The efficiency values for the ooe and oeo processes are expected to be 0.6 and 0.2 times lower than eee, respectively, according to the relative value of the involved nonlinear coefficients.

In summary, we have shown the ability of as grown SBN random quadratic medium to simultaneously achieve triply coincident nonlinear SH parametric interactions, which are continuously tunable in an extremely large spectral range $(800-1400 \mathrm{~nm})$. The spectral dependence of the SHG intensity has been theoretically modelled by considering the role of the statistical distribution of the inverted ferroelectric domains and first order QPM collinear interactions. The experimental results were found to be in good agreement with the applied model, thus providing a non-destructive alternative optical tool to other techniques such as microscopy or nonlinear spectroscopy to probe the statistical properties and the average ferroelectric domain size. ${ }^{20,22}$ Furthermore, a mutually coherent contribution of the interacting type 0 and type I nonlinear SH processes is confirmed by the theoretical fits to the experimental SHG polar plots. Additionally, the sign and relative value of the involved nonlinear coefficient for congruent SBN has been determined. In the light of this finding, the internal second harmonic interference could be exploited to obtain high contrast SH imaging of ferroelectric domain walls in SBN. Note that the extraordinary SHG vanishes when the input polarization angle is equal to $\alpha=\operatorname{arctg}\left(\sqrt{\left|\frac{d_{31}}{d_{33}}\right|}\right)$, thus providing a highly sensitive optical probe for local changes of the involved nonlinearities. Finally, the obtained results constitute a necessary step in the implementation of the recently proposed ultra-broadband entangled photon pairs from randomly poled nonlinear crys$\operatorname{tals}^{23,24}$ and increase the operative spectral bandwidth of multifunctional random quadratic media in different areas such as all optical devices, integrated optics, or polarization sensitive nonlinear sensors, with the additional advantage of a significantly simplified experimental set-up.

This work was supported by FP7 NoE Nanophotonics4Energy EU Grant No. 248855; the Spanish MICINN CSD2007-0046 (Nanolight.es), MAT2010-17443, MAT2012-31659 (SAMAP); and the Comunidad de Madrid S2009/MAT-1756 (PHAMA) projects.

${ }^{1}$ M. Baudrier-Raybaut, R. Haidar, P. Kupecek, P. Lemasson, and E. Rosencher, Nature 432, 374 (2004).

${ }^{2}$ P. Molina, M. O. Ramirez, and L. E. Bausa, Adv. Funct. Mater. 18, 709 (2008).

${ }^{3}$ A. Arie and N. Voloch, Laser Photon. Rev. 4, 355 (2010).

${ }^{4}$ R. Fischer, S. M. Saltiel, D. N. Neshev, W. Krolikowski, and Y. S. Kivshar, Appl. Phys. Lett. 89, 191105 (2006).

${ }^{5}$ M. Ayoub, P. Roedig, J. Imbrock, and C. Denz, Appl. Phys. Lett. 99, 241109 (2011).

${ }^{6}$ P. Molina, S. Alvarez-Garcia, M. O. Ramirez, J. Garcia-Sole, L. E. Bausa, H. Zhang, W. Gao, J. Wang, and M. Jiang, Appl. Phys. Lett. 94, 071111 (2009).

${ }^{7}$ R. Fischer, D. N. Neshev, S. M. Saltiel, A. A. Sukhorukov, W. Krolikowski, and Y. S. Kivshar, Appl. Phys. Lett. 91, 031104 (2007).

${ }^{8}$ P. G. Kwiat, K. Mattle, H. Weinfurter, A. Zeilinger, A. V. Sergienko, and Y. H. Shih, Phys. Rev. Lett. 75, 4337 (1995).

${ }^{9}$ X. J. Lv, J. Lu, Z. D. Xie, J. Yang, G. Zhao, P. Xu, Y. Q. Qin, and S. N. Zhu, Opt. Lett. 36, 7 (2011).

${ }^{10}$ A. Ganany-Padowicz, I. Juwiler, O. Gayer, A. Bahabad, and A. Arie, Appl. Phys. Lett. 94, 091108 (2009).

${ }^{11}$ L. Yuan, J. Shi, and X. Chen, Appl. Opt. 50, 6352 (2011).

${ }^{12}$ J. Guo, H. X. Zou, Z. H. Zhai, J. X. Zhang, and J. R. Gao, Phys. Rev. A 71, 034305 (2005).

${ }^{13}$ N. C. Menicucci, S. T. Flammia, and O. Pfister, Phys. Rev. Lett. 101, 130501 (2008).

${ }^{14}$ M. Pysher, A. Bahabad, P. Peng, A. Arie, and O. Pfister, Opt. Lett. 35, 565 (2010).

${ }^{15}$ T. Woike, T. Granzow, U. Dorfler, C. Poetsch, M. Wohlecke, and R. Pankrath, Phys. Status Solidi A 186, R13 (2001).

${ }^{16}$ M. Horowitz, A. Bekker, and B. Fischer, Appl. Phys. Lett. 62, 2619 (1993).

${ }^{17}$ W. Wang, V. Roppo, K. Kalinowski, Y. Kong, D. N. Neshev, C. Cojocaru, J. Trull, R. Vilaseca, K. Staliunas, W. Krolikowski, S. M. Saltiel, and Y. Kivshar, Opt. Express 17, 20117 (2009).

${ }^{18}$ B. F. Johnston, P. Dekker, M. J. Withford, S. M. Saltiel, and Y. S. Kivshar, Opt. Express 14, 11756 (2006).

${ }^{19}$ J. Trull, C. Cojocaru, R. Fischer, S. M. Saltiel, K. Staliunas, R. Herrero, R. Vilaseca, D. N. Neshev, W. Krolikowski, and Y. S. Kivshar, Opt. Express 15, 15868 (2007).

${ }^{20}$ M. Ayoub, P. Roedig, K. Koynov, J. Imbrock, and C. Denz, Opt. Express 21, 8220 (2013).

${ }^{21}$ F. J. Rodriguez, C. Yao, J. L. Dominguez-Juarez, J. Bravo-Abad, and J. Martorell, Opt. Lett. 36, 1347 (2011).

${ }^{22}$ Y. Sheng, A. Best, H. J. Butt, W. Krolikowski, A. Arie, and K. Koynov, Opt. Express 18, 16539 (2010).

${ }^{23}$ J. Perina, Jr. and J. Svozlik, Phys. Rev. A 83, 033808 (2011).

${ }^{24}$ J. Svozilik and J. Perina, Jr. Opt. Express 18, 27130 (2010). 